International Journal of Pure and Applied Mathematics

Volume 87 No. 4 2013, 621-628

ISSN: 1311-8080 (printed version); ISSN: 1314-3395 (on-line version)

url: http://www.ijpam.eu

doi: http://dx.doi.org/10.12732/ijpam.v87i4.11

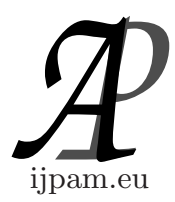

\title{
THE LAPLACIAN ON HOMOGENEOUS SPECIAL ORTHOGONAL SPACES AND THE INVERSE BRANCHING RULE
}

\author{
Liangzhong $\mathrm{Hu}^{1} \S$, José João Rossetto ${ }^{2}$ \\ ${ }^{1,2}$ Department of Mathematics \\ Federal University of Paraná \\ C.P. 019081, Curitiba, PR, 81531-990, BRAZIL
}

\begin{abstract}
The solution of the eigenvalue problem of the Laplacian with the normal metric induced by the Killing form on homogeneous special orthogonal spaces $S O(2 n+1) / S O(2 n)$ is given. The corresponding inverse branching rule from $S O(2 n)$ to $S O(2 n+1)$ is obtained.
\end{abstract}

AMS Subject Classification: 17B20, 22E46, 43A85, 81R05

Key Words: Laplacian, homogeneous space, Casimir element, highest weight, branching rule

\section{Introduction}

The solution of the eigenvalue problem of the Laplacian with the standard metric on spheres are well known in the literature. (see, for example [1] and references therein).

The Laplacian with the normal metric induced by the Killing form for semisimple compact Lie groups on homogeneous spaces (see, for example [2],[3]) plays an important role in theoretical physics, for example, in the higher dimensional quantum Hall effect [4].

Received: June 30, 2013

(C) 2013 Academic Publications, Ltd.

${ }^{\S}$ Correspondence author url: www.acadpubl.eu 
The higher dimensional quantum Hall effect involves a generalization of the Landau problem for a particle moving on homogeneous spaces, especially on $2 n$-spheres $S^{2 n}=S O(2 n+1) / S O(2 n)$ in the presence of the background gauge fields. Its further development can be found in [5], [6], [7], and [8].

A common ingredient of these analysis is the calculation of the degeneracy of the ground state for the Landau problem.

Since the Landau Hamiltonian on a homogeneous space is, up to a constant, equal to the Laplacian with the normal metric on the same homogeneous space, it is meaningful to study the solution of the eigenvalue problem of the Laplacian with the normal metric.

In [9], we gave the solution of the eigenvalue problem of the Laplacian with the normal metric on a homogeneous space $G / H$, where $G$ is a compact, semisimple Lie group, $H$ is a closed subgroup of $G$, and the rank of $H$ is equal to the rank of $G$. In [10], the solution of the eigenvalue problem of the Laplacian with the normal metric on the corresponding affine homogeneous space $\hat{g} / \hat{\boldsymbol{\eta}}$ is obtained. It is shown that the multiplicity of the lowest eigenvalue of the Laplacian with the normal metric on $G / H$ is just the degeneracy of the lowest Landau level on $G / H$, although the eigenvalues of the Laplacian are different from the Landau levels.

In this paper we apply our result to the case of $S O(2 n+1) / S O(2 n)=S^{2 n}$ and give the solution of the eigenvalue problem of the Laplacian with the normal metric. The inverse version of the branching rule from $S O(2 n)$ to $S O(2 n+1)$ is also obtained.

The layout of the paper is as follows. In Section 2, we give the eigenvalues and the multiplicities of the Laplacian on $S O(2 n+1) / S O(2 n)=S^{2 n}$. In Secction 3, the lowest eigenvalue and its multiplicity of the Laplacian on $S O(2 n+1) / S O(2 n)=S^{2 n}$ are given. Then the corresponding inverse branching rule from $S O(2 n)$ to $S O(2 n+1)$ is obtained.

\section{Eigenvalues and Multiplicities of $\Delta$ on $S O(2 n+1) / S O(2 n)$}

We first review the Laplacian on a homogeneous space $G / H$. Let $\mathbf{g}$ and $\boldsymbol{\eta}$ be the Lie algebras of $G$ and $H$, respectively. Let $U_{\mu}$ be a given irreducible representation of $\boldsymbol{\eta}$ with highest weight $\mu$. Let $G \times{ }_{H} U$ be the associated vector bundle of the principal bundle $P(G / H, H)$. The Hilbert space of square integrable sections of $G \times{ }_{H} U_{\mu}$ decomposes into the direct sum of the eigenspaces of the Laplacian on $G / H$, which are irreducible representations $V_{\lambda}$ of $\mathbf{g}$ with highest weights $\lambda$ 's. and this induces the following expression for the Laplacian 
on $G / H$ which was discussed in [11], [12], [13], [14], [15], [4], [5], [6], [7], and appears explicitly in [8].

Definition 1. The Laplacian withe normal metric induced by the Killing form for semisimple Lie groups on $G / H$ is

$$
\Delta=C_{2}(\mathbf{g}, \cdot)-C_{2}(\boldsymbol{\eta}, U) .
$$

Here $C_{2}(\mathbf{g}, \cdot)$ is the quadratic Casimir element of $\mathbf{g}$ calculated in an irreducible representation of $\mathbf{g} . C_{2}(\boldsymbol{\eta}, U)$ is the quadratic Casimir element of $\boldsymbol{\eta}$ calculated in a given irreducible representation $U$.

We take $s o(2 n)$ and $s o(2 n+1)$ be the Lie algebras of $S O(2 n)$ and $S O(2 n+1)$, respectively. We shall work in the space $\mathbf{R}^{n}$, where the inner product is the usual one and where $\epsilon_{1}, \cdots, \epsilon_{n}$ denote the usual orthonorml unit vectors which form a basis of $\mathbf{R}^{n}$. We make the usual choice of Cartan subalgebra and positive roots, $\epsilon_{i} \pm \epsilon_{j}(1 \leq i<j \leq n)$ for $s o(2 n)$ and these together with $\epsilon_{i},(1 \leq i \leq n)$ for $s o(2 n+1)$. The Weyl vectors of $s o(2 n+1)$ and $s o(2 n)$ are

$$
\rho_{s o(2 n+1)}=\left(\frac{2 n-1}{2}, \frac{2 n-3}{2}, \cdots, \frac{1}{2}\right),
$$

and

$$
\rho_{s o(2 n)}=(n-1, n-2, \cdots, 1,0) .
$$

One has

$$
\rho_{s o(2 n+1)}-\rho_{s o(2 n)}=\left(\frac{1}{2}, \cdots, \frac{1}{2}\right) .
$$

Definition 2. The Laplacian with the normal metric on $S O(2 n+1) / S O(2 n)$ is

$$
\Delta=C_{2}(s o(2 n+1), \cdot)-C_{2}(s o(2 n), U) .
$$

Here $C_{2}(s o(2 n+1), \cdot)$ is the quadratic Casimir element of $s o(2 n+1)$ calculated in an irreducible representation of $s o(2 n+1) . C_{2}(s o(2 n), U)$ is the quadratic Casimir element of $s o(2 n)$ calculated in a given irreducible representation $U$.

The spectrum of $C_{2}\left(S O(2 n+1), V_{\lambda}\right)$ in an irreducible representation $V_{\lambda}$ of so $(2 n+1)$ with highest weight $\lambda=\left(\lambda_{1}, \cdots, \lambda_{n}\right),\left(\lambda_{1} \geq \lambda_{2} \geq \cdots \geq \lambda_{n} \geq 0\right)$, is

$$
\begin{aligned}
C_{2}(\lambda) & =\left(\lambda+\rho_{s o(2 n+1)}, \lambda+\rho_{s o(2 n+1)}\right)-\left(\rho_{s o(2 n+1)}, \rho_{s o(2 n+1)}\right) \\
& =\sum_{i=1}^{n}\left[\lambda_{i}^{2}+(2 n-2 i+1) \lambda_{i}\right] .
\end{aligned}
$$

The spectrum of $C_{2}\left(S O(2 n), U_{\mu}\right)$ in a given irreducible representation $U_{\mu}$ of $s o(2 n)$ with highest weight $\mu=\left(\mu_{1}, \cdots, \mu_{n}\right),\left(\mu_{1} \geq \mu_{2} \geq \cdots \geq \mu_{n-1} \geq \mu_{n} \geq 0\right)$, 
is

$$
\begin{aligned}
C_{2}(\mu) & =\left(\mu+\rho_{s o(2 n)}, \mu+\rho_{s o(2 n)}\right)-\left(\rho_{s o(2 n)}, \rho_{s o(2 n)}\right) \\
& =\sum_{i=1}^{n}\left[\mu_{i}^{2}+2(n-i) \mu_{i}\right] .
\end{aligned}
$$

Thus we have the following result:

Theorem 3. Given an irreducible representation $U_{\mu}$ of $s o(2 n)$ with highest weight $\mu=\left(\mu_{1}, \cdots, \mu_{n}\right)$, the eigenvalue of $\Delta$ labelled by highest weight $\lambda$ reads

$$
\begin{aligned}
E_{\lambda}= & \left(\lambda+\rho_{s o(2 n+1)}, \lambda+\rho_{s o(2 n+1)}\right)-\left(\mu+\rho_{s o(2 n)}, \mu+\rho_{s o(2 n)}\right) \\
& -\left(\rho_{s o(2 n+1)}, \rho_{s o(2 n+1)}\right)+\left(\rho_{s o(2 n)}, \rho_{s o(2 n)}\right) \\
= & \sum_{i=1}^{n}\left[\lambda_{i}^{2}-\mu_{i}^{2}+(2 n-2 i+1) \lambda_{i}-2(n-i) \mu_{i}\right] .
\end{aligned}
$$

The multiplicity of the eigenvalue $E_{\lambda}$ is given by the Weyl dimension formula:

$$
\begin{aligned}
\operatorname{dim} V_{\lambda}= & \frac{\prod_{\alpha \in \Phi_{\mathrm{so}(2 \mathrm{n}+1)}^{+}}\left(\lambda+\rho_{s o(2 n+1)}, \alpha\right)}{\prod_{\alpha \in \Phi_{\mathrm{so}(2 \mathrm{n}+1)}^{+}\left(\rho_{s o(2 n+1)}, \alpha\right)}} \\
= & \prod_{i=1}^{n} \frac{2 \lambda_{i}+2 n+1-2 i}{2 n+1-2 i} \\
& \times \prod_{1 \leq i<j \leq n} \frac{\left(\lambda_{i}-\lambda_{j}+j-i\right)\left(\lambda_{i}+\lambda_{j}+2 n+1-i-j\right)}{(j-i)(2 n+1-i-j)} .
\end{aligned}
$$

The above highest weights $\lambda$ 's will be determined by Theorem 5 in the next section.

\section{The Multiplicity of the Lowest Eigenvalue of $\Delta$ and the Inverse Branching Rule}

Let us determine the multiplicity of the lowest eigenvalue of $\Delta$, we first review the relation between the Weyl groups $W_{s o(2 n+1)}$ of $s o(2 n+1)$ and $W_{s o(2 n)}$ of $s o(2 n)$, where $W_{s o(2 n)}$ is a subgroup of $W_{s o(2 n+1)}$ [16]. Choose the positive roots consistently. Then the positive Weyl chamber $\mathcal{W}_{s o(2 n+1)}$ of $\operatorname{so}(2 n+1)$ is contained in the positive Weyl chamber $\mathcal{W}_{s o(2 n)}$ of $s o(2 n)$. The interior of positive Weyl chamber for $s o(2 n+1)$ consists of all

$$
x=\left(x_{1}, \cdots, x_{n}\right)=x_{1} \epsilon_{1}+\cdots+x_{n} \epsilon_{n}
$$

with

$$
x_{1}>x_{2}>\cdots>x_{n}>0 \text {, }
$$


whereas the interior of positive Weyl chamber of $s o(2 n)$ consists of all $x$ satisfying

$$
x_{1}>x_{2}>\cdots>x_{n-1}>\left|x_{n}\right| .
$$

Let

$$
C \subset W_{s o(2 n+1)}
$$

denote the set of elements that map $\mathcal{W}_{s o(2 n+1)}$ into $\mathcal{W}_{s o(2 n)}$. Then

$$
C=\{e, s\} .
$$

Here $e$ is the identity and $s$ is the reflection, which changes the sign of the last coordinate.

$$
\mathcal{W}_{s o(2 n)}=\mathcal{W}_{s o(2 n+1)} \bigcup s \mathcal{W}_{s o(2 n+1)},
$$

while

$$
W_{s o(2 n+1)}=W_{s o(2 n)} \cdot C .
$$

Given an irreducible representation $U_{\mu}$ of $s o(2 n)$ with highest weight $\mu=$ $\left(\mu_{1}, \cdots, \mu_{n}\right),\left(\mu_{1} \geq \mu_{2} \geq \cdots \geq \mu_{n-1} \geq \mu_{n} \geq 0\right)$, it can be found that $\mu$ corresponds to a unique highest weight $\lambda$ of an irreducible representation $V_{\lambda}$ of so $(2 n+1)$ :

$$
\begin{aligned}
\lambda & =\mu+\rho_{s o(2 n)}-\rho_{s o(2 n+1)} \\
& =\left(\mu_{1}-\frac{1}{2}, \cdots, \mu_{n}-\frac{1}{2}\right) .
\end{aligned}
$$

It follows that

$$
\left(\lambda+\rho_{s o(2 n+1)}, \lambda+\rho_{s o(2 n+1)}\right)=\left(\mu+\rho_{s o(2 n)}, \mu+\rho_{s o(2 n)}\right) .
$$

By theorem 3, the lowest eigenvalue of $\Delta$ is

$$
E_{\left(\mu_{1}-\frac{1}{2}, \cdots, \mu_{n}-\frac{1}{2}\right)}=\left(\rho_{s o(2 n)}, \rho_{s o(2 n)}\right)-\left(\rho_{s o(2 n+1)}, \rho_{s o(2 n+1)}\right) .
$$

Thus we have the following result:

Theorem 4. Given an irreducible representation $U_{\mu}$ of so $(2 n)$ with highest weight $\mu=\left(\mu_{1}, \cdots, \mu_{n}\right),\left(\mu_{1} \geq \mu_{2} \geq \cdots \geq \mu_{n-1} \geq \mu_{n} \geq 0\right)$, the eigenspace of the lowest eigenvalue of $\Delta$ on $S O(2 n+1) / S O(2 n)$ is $V_{\lambda}$ with highest weight $\lambda=\left(\mu_{1}-\frac{1}{2}, \cdots, \mu_{n}-\frac{1}{2}\right)$. The lowest eigenvalue of $\Delta$ is

$$
\begin{aligned}
E_{\left(\mu_{1}-\frac{1}{2}, \cdots, \mu_{n}-\frac{1}{2}\right)} & =\left(\rho_{s o(2 n)}, \rho_{s o(2 n)}\right)-\left(\rho_{s o(2 n+1)}, \rho_{s o(2 n+1}\right) \\
& =\frac{1}{4} n-\frac{1}{2} n^{2} .
\end{aligned}
$$


The multiplicity of the lowest eigenvalue of $\Delta$ is

$$
\begin{aligned}
\operatorname{dim}_{\left(\mu_{1}-\frac{1}{2}, \cdots, \mu_{n}-\frac{1}{2}\right)} & =\prod_{i=1}^{n} \frac{2 \mu_{i}+2 n-2 i}{2 n+1-2 i} \\
\times & \prod_{1 \leq i<j \leq n} \frac{\left(\mu_{i}-\mu_{j}+j-i\right)\left(\mu_{i}+\mu_{j}+2 n-i-j\right)}{(j-i)(2 n+1-i-j)} .
\end{aligned}
$$

Remark. It should be mentioned that $\lambda=\mu+\rho_{s o(2 n)}-\rho_{s o(2 n+1)}$ is the highest weight of the lowest eigenvalue of $\Delta$ (the ground Landau level). In the literature, this highest weight was often taken to be $\lambda=\mu$.

Notice that the eigenvalues of $\Delta$ are different from the Landau levels due to the different evaluation of the quadratic Casimir element $C_{2}\left(S O(2 n), U_{\mu}\right)$.

By Theorem 4 and the branching rule from $S O(2 n+1)$ to $S O(2 n)$ [17], we have

Theorem 5. Given an irreducible representation $U_{\mu}$ of $s o(2 n)$ with highest weight $\mu=\left(\mu_{1}, \cdots, \mu_{n}\right),\left(\mu_{1} \geq \mu_{2} \geq \cdots \geq \mu_{n-1} \geq \mu_{n} \geq 0\right)$, the highest weights appeared in Theorem $3, \lambda=\left(\lambda_{1}, \cdots, \lambda_{n}\right)$ with $\left(\lambda_{1} \geq \lambda_{2} \geq \cdots \geq \lambda_{n} \geq 0\right)$ satisfy the following inequalities:

$$
\begin{gathered}
\left(\lambda+\rho_{s o(2 n+1)}, \lambda+\rho_{s o(2 n+1)}\right) \geq\left(\mu+\rho_{s o(2 n)}, \mu+\rho_{s o(2 n)}\right) . \\
\lambda_{1}+\frac{1}{2} \geq \mu_{1} \geq \lambda_{2}+\frac{1}{2} \geq \mu_{2} \geq \cdots \geq \lambda_{n-1}+\frac{1}{2} \geq \mu_{n-1} \geq \lambda_{n}+\frac{1}{2} \geq \mu_{n} \geq 0 .
\end{gathered}
$$

The Hilbert space of square integrable sections of the associated vector bundle $S O(2 n+1) \times_{S O(2 n)} U_{\mu}$ of the principal bundle $P(S O(2 n+1) / S O(2 n), S O(2 n))$ decomposes into the direct sum of the eigenspaces of the Laplacian on $S O(2 n+$ 1) $/ S O(2 n)$, which are irreducible representations $V_{\lambda}$ of $s o(2 n+1)$ with the above highest weights $\lambda$ 's.

Theorem 5 can be considered as an inverse version of the branching rule from $S O(2 n)$ to $S O(2 n+1)$.

\section{References}

[1] M. A. Shubin, Pseudodifferential Operators and Spectral Theory, SpringerVerlag (2001).

[2] N. R. Wallach, Harmonic Analysis on Homogeneous Spaces, Dekker (1973).

[3] S. Helgason, Differential Geometry, Lie Groups and Symmetric Spaces, Academic Press (1978). 
[4] S.-C. Zhang and J. Hu, A four dimensional generalization of the quantum Hall effect, Science, 294 (2001), 823-828. DOI: 10.1126/science.294.5543.823

[5] D. Karabali and V. P. Nair, Quantum Hall effect in higher dimensions, Nucl. Phys. B, 641 (2002), 533-546. DOI:10.1016/S0550-3213(02)00634-X

[6] B. Dolan, The spectrum of the Dirac operator on coset spaces with homogeneous gauge fields, JHEP, 0305 (2003), 018. DOI:10.1088/1126$6708 / 2003 / 05 / 018$

[7] B. A. Bernevig, J. Hu, N. Toumbas and S.-C. Zhang, The eight dimensional quantum Hall effect and the octonions, Phys. Rev. Lett. 91 (2003), 236803236807. DOI:10.1103/PhysRevLett.91.236803

[8] G. Meng, Geometric construction of the Quantum Hall Effect in all even dimensions, J. Phys. A, 369415 (2003), 9415-9424. DOI:10.1088/0305$4470 / 36 / 36 / 301$

[9] L. Hu, The Laplacian on homogeneous spaces, J. Math. Phys., 49 (2008), 053513. DOI:10.1063/1.2924268

[10] L. Hu and J. J. Rossetto, The Laplacian on an affine homogeneous space, Int. J. Pure and Appl. Math. 85 (2013), 531-539. DOI:10.12732/ijpam.v85i3.8

[11] C. N. Yang, Generalization of Diracs monopole to $S U_{2}$ gauge fields, $J$. Math. Phys., 19 (1978), 320-328. DOI:10.1063/1.523506

[12] M. Stone, Supersymmetry and the quantum mechanics of spin, Nucl. Phys. $B, 314$ (1989), 557-586. DOI:10.1016/0550-3213(89)90408-2

[13] V. D. Lyakhovsky, N. N. Shtykov and D. V. Vassilevich, DeWitt-Schwinger expansion for projective and Grassmann spaces, Lett. Math. Phys., 21 (1991), 89-95. DOI:10.1007/BF00401641

[14] N. P. Landsman, Induced representations, gauge fields, and quantization on homogeneous spaces, Rev. Math. Phys., 4 (1992), 503-528. DOI: 10.1142/S0129055X92000212

[15] P. Lévay, D. McMullan and I.Tsutsui, The canonical connection in quantum mechanics, J. Math. Phys., 37 (1996), 625-636. DOI:10.1063/1.531432 
[16] B. Gross, B. Kostant, P. Ramond and S. Sternberg, The Weyl character formula, the half-spin representations, and equal rank subgroups, Proc. Natl. Acad. Sci. USA, 95 (1998), 8441-8442. DOI:10.1073/pnas.95.15.8441

[17] R. Goodman and N. Wallach, Representations and Invariants of the Classical Groups, Encyclopedia Math. Appl. 68, Cambridge University Press (1998). 